\title{
Dosage automatique de l'urée dans l'eau de mer : une méthode très sensible à la diacétylmonoxime
}

\author{
A. Aminot et R. Kerouel
}

\author{
CNEXO, Centre Océanologique de Bretagne, BP 337, 29273 Brest Cedex, France
}

\begin{abstract}
Aminot, A., ET R. KEROuel. 1982. Dosage automatique de l'urée dans l'eau de mer : une méthode très sensible à la diacétylmonoxime. Can. J. Fish. Aquat. Sci. 39: 174-183.
\end{abstract}

\begin{abstract}
-Une méthode automatique, basée sur la réaction avec la diacétylmonoxime, a êté mise au point pour le dosage de l'urée dans l'eau de mer. L'utilisation de réactifs contenant de la thiosemicarbazide et du fer ferrique a permis une augmentation importante de la sensibilité par rapport aux méthodes existantes. La sensibilité est de 0,044 unité d'absorbance par $\mu$ atg-N-urée/ $\mathrm{L}$ pour $50 \mathrm{~mm}$ de trajet optique. La reproductibilité est de $\pm 0,01 \mu$ atg- $\mathrm{N}$-urée/ $\mathrm{L}$ et la limite de détection (2 écarts-types) de $0,02 \mu$ atg- $\mathrm{N}$-urée/L. La relation absorbanceconcentration est linéaire jusqu'à $15 \mu \mathrm{atg}-\mathrm{N}$-urée/L. Les blancs analytiques ont été déterminés rigoureusement et les interférences étudiées. La citrulline est la principale interférence. Toutefois, en utilisant une méthode de chélation sur résine, pour éliminer ce composé des échantillons, nous avons pu démontrer son absence dans les eaux côtières analysées. La salinité $n$ 'affecte pas la réaction avec l'urée entre 0 et $35 \%$. Les problèmes de contamination et de conservation ont été envisagés : de grandes précautions doivent être prises pour la manipulation des échantillons; la congélation rapide s'est révélée efficace pour une longue conservation. Quelques exemples d'applications sont présentés en milieux estuarien, côtier et

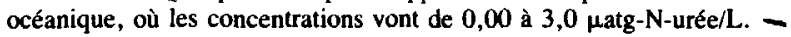

Mots clefs: urée, eau de mer, estuaires, méthode automatique.

Aminot, A., ET R. Kerouel. 1982. Dosage automatique de l'urée dans l'eau de mer : une méthode très sensible à la diacétylmonoxime. Can. J. Fish. Aquat. Sci. 39: $174-183$.

- The determination of urea in sea water was performed by an automated method based on the reaction of urea with diacetyl-monoxime. The sensitivity, which has been greatly enhanced compared to previous methods, by using reagents containing thiosernicarbazide and iron III, is 0.044 absorbance units $/ \mu \mathrm{g}$-at urea- $\mathrm{N} / \mathrm{L}$ with the $50 \mathrm{~mm}$ flow cell. The precision is $\pm 0.01 \mu \mathrm{g}$-at urea- $\mathrm{N} / \mathrm{L}$ and the limit of detection (2 SD) is $0.02 \mu \mathrm{g}$-at urea-N/L. The absorbance - concentration relationship is linear up to $15 \mu \mathrm{g}$-at urea-N/L. The analytical blanks have been strictly determined and the interferences studied. Citrulline is the main compound that interferes. However, in attempting to remove this compound from the samples by a chelating resin, we demonstrated its absence from the analyzed coastal waters. The color development with urea does not depend on the salt concentration. The problems of contamination and storage are discussed: great care must be taken in handling the samples; quickly freezing the samples is proved very suitable for storage during a long time. Some examples of application of the method to oceanic, coastal and estuarine waters show concentrations ranging from 0.00 to $3.0 \mu \mathrm{g}$-at urea-N/L.

Reçu le 23 avril 1980

Accepté le 11 septembre 1981

LES premières observations sur l'utilisation de l'urée par certaines espèces du phytoplancton ont été faites par Dugdale et Goering (1967) et McCarthy (1970). Corner et Newell (1967) et Newell et al. (1967) mettaient en évidence l'excrétion d'urée par le zooplancton. L'importance de l'urée dans le cycle de l'azote en milieu marin était ainsi démontrée et a fait l'objet de divers travaux sur l'assimilation par le phyto-

'Contribution $n^{\circ} 752$ du Centre Océanologique de Bretagne.

Printed in Canada (J6046)

Imprimé au Canada (J6046)
Received April 23, 1980 Accepted September 11, 1981 plancton, parmi lesquels on peut citer principalement ceux de Eppley et al. (1971), McCarthy (1972), Carpenter et al. (1972) et Antia et al. (1977).

Par ailleurs, l'urée est un des principaux composés azotés excrétés par les mammifères et, à ce titre, peut s'avérer être, en eaux côtières, un traceur des rejets de type agricole ou urbain (11,2 $\mu \mathrm{atg}-\mathrm{N}$-urée/L dans le port de New-York; Remsen 1971). Certain effluents industriels contiennent également de l'urée en forte concentration, celle-ci pouvant alors être considérée comme un polluant (Mauzac et al. 1976).

Pour toutes ces applications, le dosage de l'urée présente un 
intérêt certain, et il doit à la fois être simple, sensible et dépourvu d'interférences.

Les premières méthodes se sont développées en chimie clinique et en biochimie (analyses d'urine et de sang) pour le dosage de l'urée ou des dérivés uréidiques (citrulline, acide carbamylaspartique). On peut les classer en deux catégories : la méthode enzymatique et la méthode colorimétrique. La première méthode consiste à transformer quantitativement l'urée en ammonium en présence d'uréase, puis à doser l'ammonium par une méthode classique, titrimétrique ou colorimétrique. Mise au point par Marshall (1913). pour l'analyse de l'urée dans l'urine. la méthode enzymatique a été développée et améliorée progressivement. Son application à l'analyse de l'urée dans l'eau de mer a été réalisé par McCarthy (1970) et reprise par ailleurs dans le manuel d'analyse de Strickland et Parsons (1972).

La méthode colorimétrique directe est basée sur. la formation d'un composé coloré par réaction de l'urée avec la diacétylmonoxime. La première application à l'eau de mer de la méthode à la diacétylmonoxime a été réalisée par Newell et al. (1967) d'après Beale et Croft (1961). Cette méthode manuelle a été adaptée à l"analyse automatique par DeManche et al. (1973) qui concluent à sa supériorité sur la méthode à l'uréase : plus grande simplicité pour une précision comparable.

Pour l'analyse des eaux naturelles, on peut également citer la méthode au phénol-hypochlorite de Emmet (1969) qui n'a pas d'antécédent clinique. Cette méthode ne parait pas avoir eté largement utilisée, vraisemblablement en raison de son manque de sensibilité

Pour notre part, après avoir tout d'abord appliqué la méthode de DeManche et al. (1973), nous avons constaté qu'elle manquait de sensibilité pour l'analyse des eaux naturelles et principalement des eaux de mer. Notre objectif a été d'accroitre la sensibilité de la méthode colorimétrique afin de pouvoir analyser l'urée en milieu marin avec un meilleure fuabilité. Parallèlement, il était souhaitable de réduire la complexité des réactifs tout en maintenant la qualité analytique maximale. Nous avons pu satisfaire à ces exigences grâce à des modifications importantes des réactifs et à la réduction de la température de réaction. éliminant ainsi les inconvénients majeurs de la méthode colorimétrique directe.

\section{Principe Général}

La méthode colorimétrique directe a pour origine les travaux de Fearon (1939) qui obtint la formation de produits colorés jaunes par condensation en milieu fortement acide et oxydation de la diacétylmonoxime avec les composés du type $R_{1}-\mathrm{NH}-\mathrm{CO}-\mathrm{NHR}_{2}$ ( $R_{1}$ est un radical alkyle ou un hydrogène; $R_{2} n$ 'est pas un radical acyle). Un nombre tres important de travaux ont été réalisés jusqu'à ce jour concernant l'application de cette réaction au dosage de l'urée (où $R_{1}=R_{2}=H$ ) ou de dérivés uréidiques en chimie clinique. Comme le soulignent Beale et Croft (1961), cet aussi grand nombre de travaux témoigne de l'ampleur des difficultés à résoudre : instabilité des colorations à la lumière ou dans le temps, faible sensibilité, température de réaction élevée, non linéarité des courbes d'étalonnage (Ceriotti et Spandrio 1963). Cependant. des améliorations ont été apportées en faisant intervenir dans la réaction un nombre croissant de composés destinés à remé- dier aux principaux défauts observés. La présence de molécules organiques particulières, aboutissant à la formation d'un composé rouge, a permis l'accroissement de la sensibilité : acide phénylanthranilique (Wheatley 1948; Beale et Croft 1961). phénazone (Ceriotti et Spandrio 1963; Prescott et Jones 1969), semicarbazide (Newell et al. 1967; DeManche et al. 1973), thiosemicarbazide (Coulombe et Favreau 1963; Marsh et al. 1965).

Diverses combinaisons d'acides, d'oxydants. de catalyseurs (ions métalliques) peuvent améliorer la stabilité de la coloration ou la vitesse de réaction. Cependant la complexité et la diversité des milieux réactionnels entraine parfois la publication de résultats contradictoires. Alors que Fearon (1939), par exemple, emploie un oxydant, Beale et Croft (1961) le jugent inutile sinon nuisible. De même, le cuivre, considéré comme un stabilisant efficace par ces derniers auteurs, inhibe la réaction de Ceriotti et Spandrio (1965).

\section{Matériel et Réactifs}

\section{APPAREILLAGE}

Nous avons utilisé une chaîne d'analyse automatique Technicon Autoanalyzer II. avec un échantillonneur de type Sampler IV , une pompe modèle P III et un colorimètre SCIC équipé d'une cuve de $50 \mathrm{~mm}$ de trajet optique et de filtres interférentiels à $520 \mathrm{~nm}$. Deux types de bains-marie ont été essayés avec succès : d'une part un bain-marie Technicon AAl, d autre part un bain-marie Chemlab CHB 40 (bobines: longueur $=13 \mathrm{~m}$. diamètre intérieur $=1.6 \mathrm{~mm}$ ). Tout autre bain-marie doit pouvoir convenir s'il peut être équipé d'une bobine de délai permettant un temps de chauffage de $10 \mathrm{~min}$ au moins, et si les fluctuations de température ne dépassent pas $\pm 0,2^{\circ} \mathrm{C}$ à environ $90^{\circ} \mathrm{C}$.

\section{REACTIFS}

Les produits utilisés sont tous de qualité « pour analyse". - Solution de thiosemicarbazide : dissoudre $0.95 \mathrm{~g}$ de thiosemicarbazide $\left(\mathrm{H}, \mathrm{N}-\mathrm{NH}-\mathrm{CS}-\mathrm{NH}_{2}\right)$ dans $100 \mathrm{~mL}$. d'eau distillée. Cette solution se conserve plusieurs mois en flacon de verre à température ambiante.

- Solution ferrique : dissoudre $0.15 \mathrm{~g}$ de chlonure ferrique $\left(\mathrm{FeCl}_{3}, 6 \mathrm{H}_{2} \mathrm{O}\right)$ dans $10 \mathrm{~mL}$ d'eau distillée.

- Réactif $\mathrm{A}$ : dissoudre $8.5 \mathrm{~g}$ de diacétylmonoxime dans $250 \mathrm{~mL}$ d'eau distillée portée à $80^{\circ} \mathrm{C}$. Refroidir à température ambiante, puis ajouter $10 \mathrm{~mL}$ de solution de thiosemicarbazide. Placé à l'abri de la lumière ce réactif est stable une semaine, en flacon de verre, à température ambiante.

- Réactif B : verser avec précaution $300 \mathrm{~mL}$ d'acide sulfurique (densité $=1.84$ ) dans $235 \mathrm{~mL}$ d'eau distillée. Après refroidissement ajouter $0,5 \mathrm{~mL}$ de la solution de chlorure ferrique et homogénéiser. Ce réactif se conserve indéfiniment.

- Solution étalon d'urée : dans un litre d'eau distillée. dissoudre $150.2 \mathrm{mg}$ d'urée préalablement séchée à $100^{\circ} \mathrm{C}$ pendant une heure. Ajouter quelques gouttes de chloroforme. Conservée au réfrigérateur. cette solution est stable pendant plusieurs mois. La concentration de cette solution est de 5 ratg-N-urée $/ \mathrm{mL}$ (ou $2,5 \mu \mathrm{mol} / \mathrm{mL}$ ). 


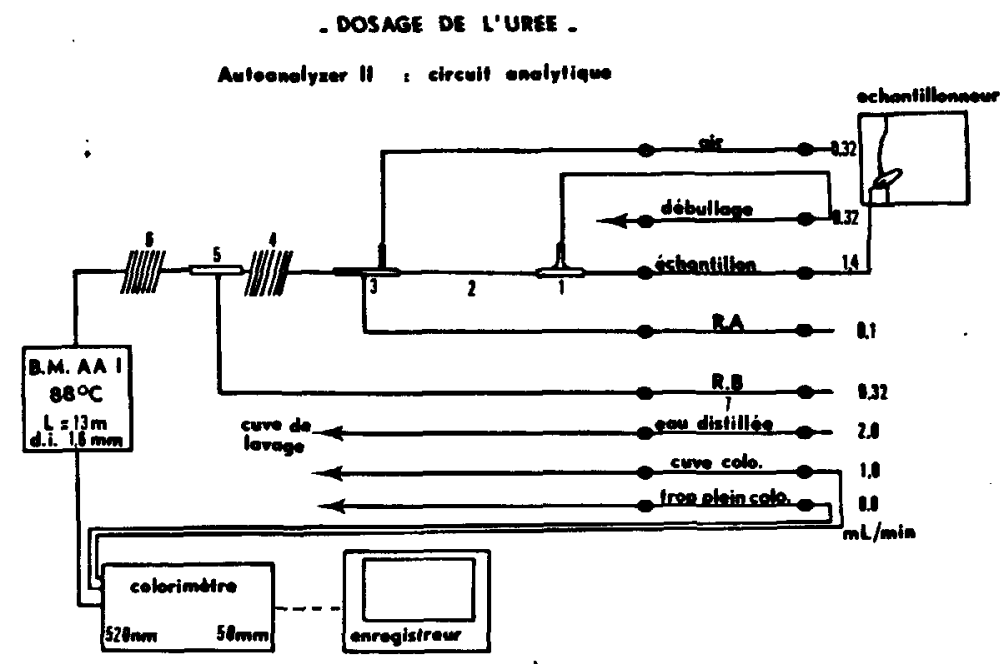

Fig. I. Circuit analytique pour l'analyse automatique de l'urée. 1 : débulleur (Technicon 116-0202-00); 2 : emplacement pour une colonne éventuelle de Chelex 100 (voir texte); 3 : injecteur double (Technicon 116-0489-01E); 4 et 6 : bobines de délai, 20 spires; 5 : injecteur verre (Technicon DI 116-0203-01); 7 : tube acidflex.

\section{Mode opératoire}

\section{CiRCUTT ANALYTIQUE}

Le circuit analytique est représenté sur la fig. 1. On notera la présence inhabituelle d'un repompage du trop plein de la cuve du colorimètre. Ce repompage s'est avéré nécessaire pour une parfaite régulation du flux qui, sans cet artifice, présentait des pulsations engendrant une oscillation de la ligne de base. Nous attribuons ce phénomène à la présence du long bain-marie à $88^{\circ} \mathrm{C}$. dans lequel la réaction s'effectue pendant $11 \mathrm{~min}$.

Les réactifs A et B peuvent être introduits dans un ordre quelconque sans que les résultats en soient modifiés.

Le lavage du circuit entre les échantillons est effectué par de l'eau distillée. En fin de journée un rinçage à l'eau distillée suffit également à assurer le nettoyage du circuit.

Le rythme d'analyse habituel est de 20 échantillons à l'heure, avec un temps de prélèvement de 1 min et un temps de lavage de 2 min. Des essais à 30 échantillons à l'heure ont également donné toute satisfaction.

\section{EAUX DE RÉFÉRENCE ET BLANC D'EFFET DE SEL}

Lors de toute analyse de trace, il est important de disposer d'eaux de référence dans lesquelles la concentration de l'espèce recherchée est nulle. Sans cette précaution les mesures seront entachées d'erreurs systématiques.

Pour les eaux son salées, nous n'avons pas mesuré de différence de signal entre de l'eau distillée. de l'eau déminéralisée après distillation et de l'eau bidistillée en milieu oxydant. On peut donc considérer que de l'eau correctement distillée ne contient normalement pas d'urée et convient comme référence. Il est cependant souhaitable de la contrôler

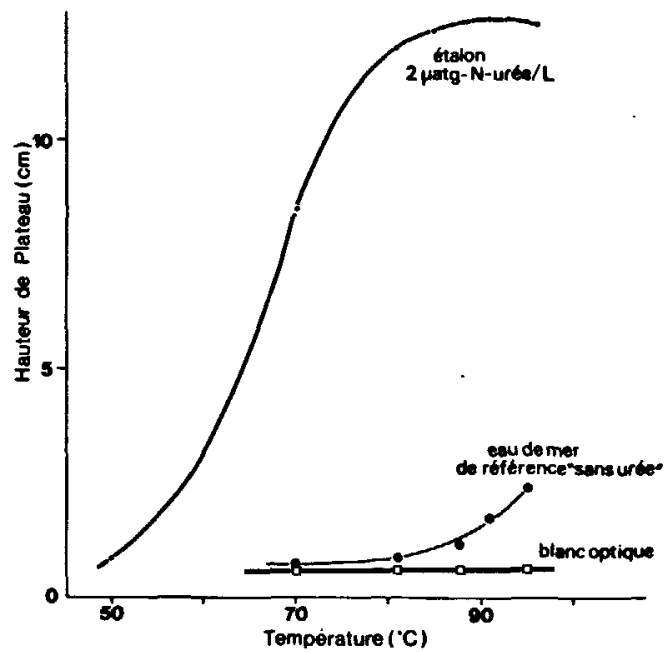

Fic. 2. Influence de la température sur la coloration $(\cdot)$, le blanc d'effet de sel 1 ) et le blanc optique ([).

par rapport à une eau déminéralisée ou bidistillée.

Dans le cas de l'analyse d'eaux marines, on ne peut utiliser l'eau distillée comme référence de zéro de concentration en raison de la présence d'un blanc dû à un effet de sel. Lorsque nous inhibons totalement le développement de la coloration en remplaçant le réactif à la diacétylmonoxime par de l'eau distillée, le passage d'un échantillon d'eau de mer ( $S=35 \%$ ) se traduit par un signal qui, mesuré par rapport à l'eau distillée, équivaut à $0,12 \mu$ atg- $\mathrm{N}$-urée/L. Ce signal ne varie pas 


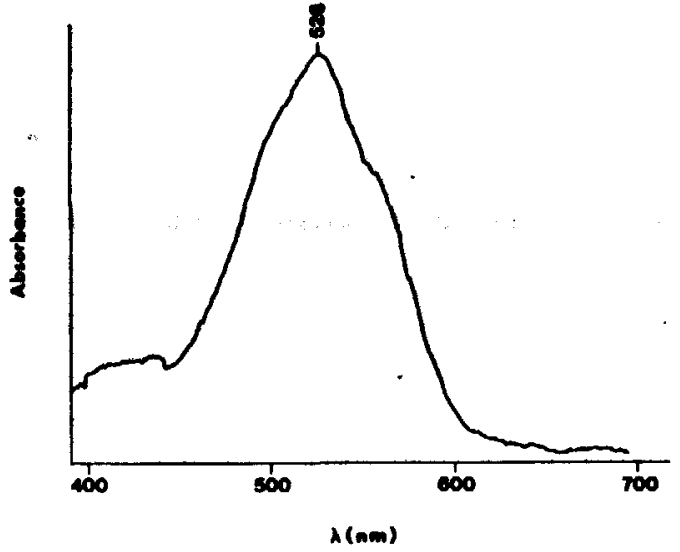

Fig. 3. Spectre d'absorption du composé formé au cours de la réaction de l'urée avec la diacétylmonoxime en présence de thiosemicarbazide.

avec la température (fig. 2). c'est un «blanc optique » lié à la configuration du système optique du colorimètre (Froelich et Pilson 1978). En outre, les mesures réalisées avec tous les réactifs sur une eau océanique profonde, à teneur négligeable en urée, montrent une croissance très rapide du signal (mesuré par rapport à l'eau distillée) pour les températures supérieures à $90^{\circ} \mathrm{C}$ (fig. 2). Comme la température influe très peu sur la hauteur d'un ajout dans l'eau de mer entre 85 et $95^{\circ} \mathrm{C}$ (fig. 2), la présence de traces éventuelles d'urée ne peut donc être à l'origine de cette croissance rapide du signal. Une réaction parasite qui ne fait pas intervenir l'urée se produit donc en milieu salin aux températures élevées. La détermination rigoureuse du blanc d'effet de sel total (blanc optique + réaction parasite) se fera donc par analyse d'une eau de mer exempte d'urée.

Dans ce but, et par analogie avec l'ammonium (Le Corre et Tréguer 1978), nous avons cherché à obtenir une référence absolue en analysant des eaux de mer prélevées par grandes profondeurs dans le Golfe de Gascogne. Le signal, mesuré pour de l'eau de $3000 \mathrm{~m}(\mathrm{~S}=35 \%)$, représente un minimum absolu de $0,17 \mu$ atg- $\mathrm{N}$-urée $/ \mathrm{L}$ à $88^{\circ} \mathrm{C}$. Par rapport au «blanc optique» cette valeur correspond à $0.05 \mu$ atg- $N$ urée/L. Après passage de cette eau de mer sur colonne de granulés de charbon actif $(0,6 \mathrm{~cm} \times 35 \mathrm{~cm} ; 1,5 \mathrm{~mL} / \mathrm{min})$. le signal ne montre aucune baisse contrairement à ce que l'on observe avec un étalon d'urée. Nous pouvons par conséquent attribuer raisonnablement la concentration $0.00 \mu \mathrm{\mu tg}-\mathrm{N}$ urée $/ \mathrm{L}$ à cette eau de mer prélevée à $3000 \mathrm{~m}$ (fig. 7).

Une autre technique, également similaire à celle pratiquée pour lammonium (Grasshoff 1976), permet d'obtenir de l'eau de mer extrèmement pauvre en urée. Si on laisse stagner, dans un baril de polyéthylène. à la lumière et à la température du laboratoire, de l'eau de mer filtrée à $200 \mu \mathrm{m}$ et peu concentrée en urée. ce composé se dégrade presque totalement. Nous avons obtenu de cette façon des concentrations résiduelles de $0,09 \mu \mathrm{atg}-\mathrm{N}$-urée/L. Par passage sur charbon actif, comme précédemment, la concentration s'est abaissée à une valeur très voisine de celle de l'eau profonde

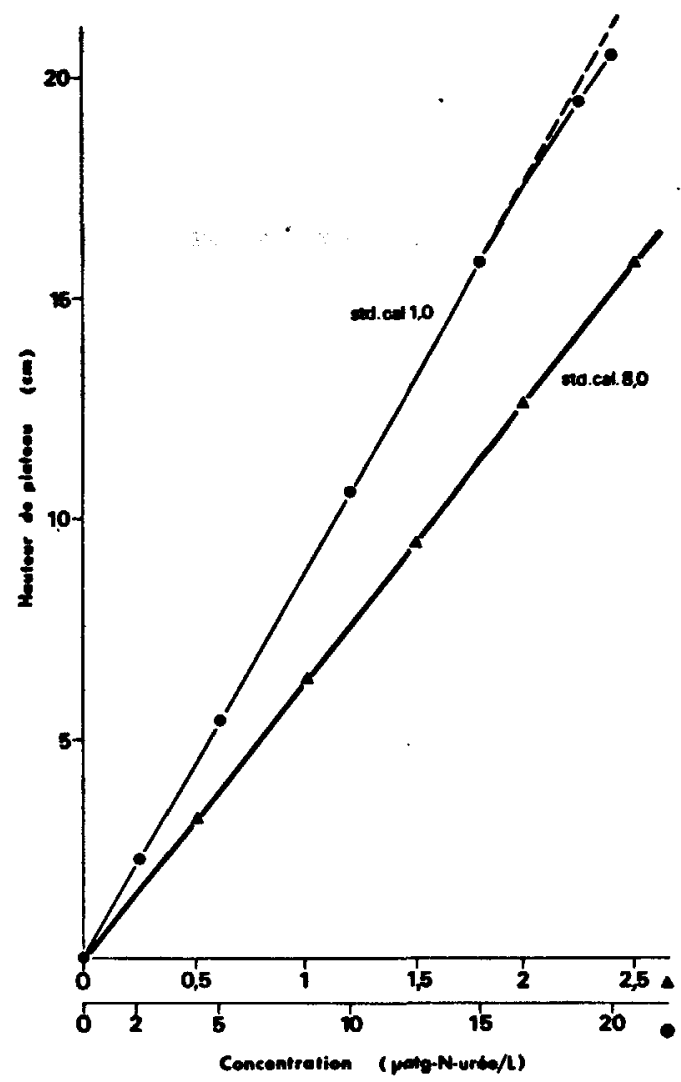

FiG. 4. Etalonnages pour l'analyse de I'uree en eau de mer. $\Delta$

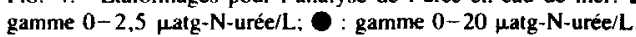

\section{( $<0.02 \mu$ atg-N-uré/L).}

Ce moyen simple d'obtention d'eau de mer de référence exempte d'urée permet de mesurer avec rigueur le blanc d'effet de sel total pour une salinité déterminée. Cet effet étant proportionnel à la salinité, il se calcule aisément pour une eau de mer de salinité quelconque connue. Le blanc d'effet de sel total dépendant essentiellement du blanc optique $(70 \%$ dans le cas présent) il doit être déterminé avec chaque colorimètre utilisé.

\section{PRECAUTIONS}

Les contaminations des échantillons peuvent être importantes si l'on ne prend pas un certain nombre de précautions.

Tout contact avec les doigts devra être évité et il est conseillé d'utiliser des gants pour la manipulation des échantillons. en particulier pour leur transfert dans les godets de l'Autoanalyzer (Ceriotti et Spandrio 1965). L'utilisation d'un échantillonneur sur lequel on peut placer directement les flacons sans transvasage est. à ce point de vue, préférable. It faut également éviter l'utilisation de flacons comportant une capsule d'étanchéité que l'on introduit dans le goulot. 


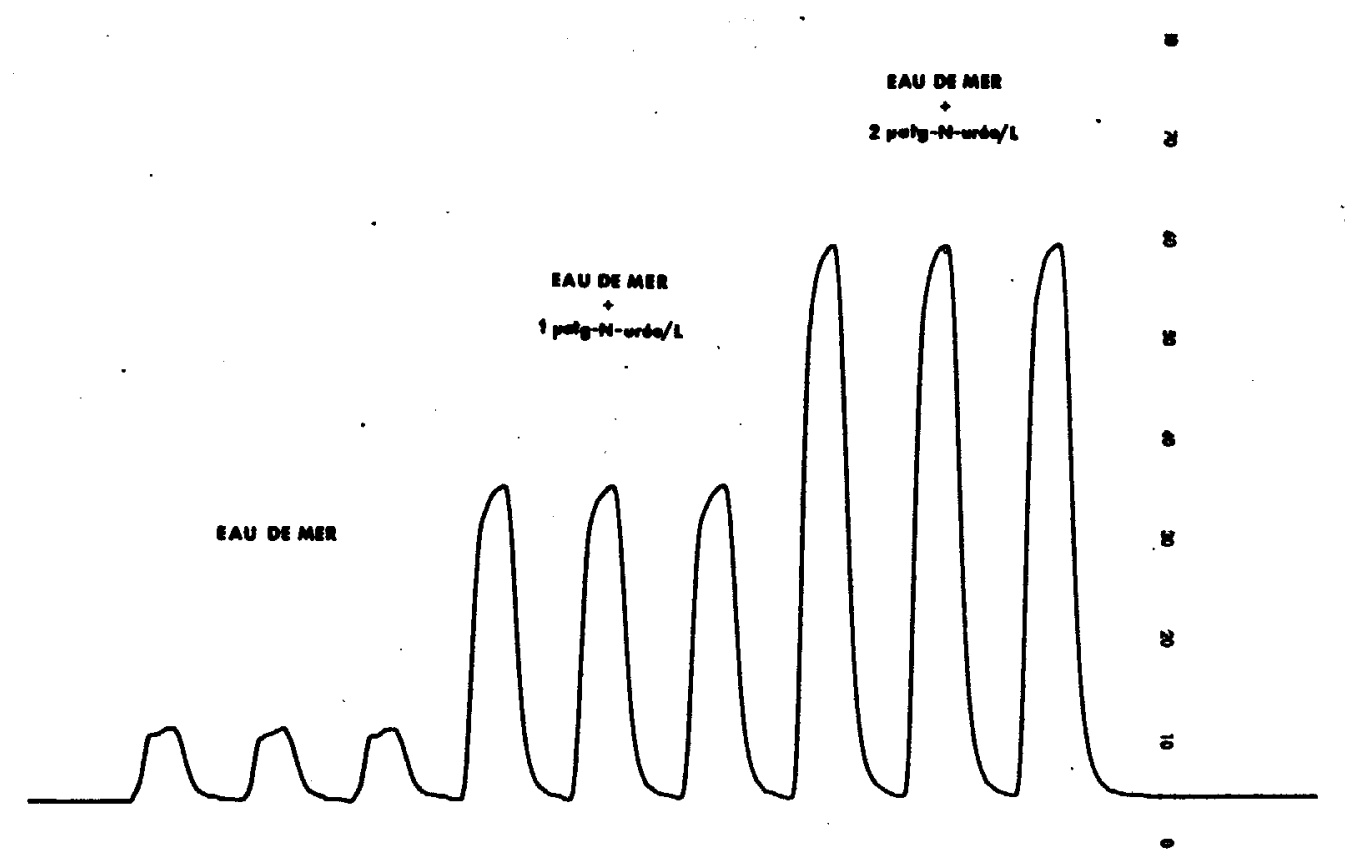

FIG: 5. Enregistrement d'une série de trois groupes de pics correspondant à une eau de mer pauvre en urée et à cette même eau de mer additionné de I et $2 \mu$ atg- $\mathrm{N}$-urée/L. Fréquence : 20 échantillons/h; prélèvement/lavage $: 1 \mathrm{~min} / 2 \mathrm{~min} ;$ cuve : $50 \mathrm{~mm} ; \mathrm{Std}$. Cal. : 8,0.

Si l'eau contient plus de quelques milligrammes par litre de particules en suspension, la turbidité qui en résulte devient détectable optiquement et il faut l'éliminer. Cependant, la filtration peut accroître les risques de contamination, en particulier par la présence d'urée dans certains filtres. Dans ce but. Koroleff (dans Grasshoff 1976) préconise la centrifugation des échantillons les plus turbides. Pour notre part nous avons testé des filtres Whatman $\mathrm{GF} / \mathrm{C}$ portés une heure à $500^{\circ} \mathrm{C}$ et montés sur un support filtre pour seringue en acier inoxydable (Millipore). Cet ensemble n'a apporté aucune contamination des échantillons.

Quant aux problèmes de prélèvement, nous avons parfois noté, comme le signale Koroleff (dans Grasshoff 1976). des contaminations des eaux de surface par les rejets du bateau (cuisine, toilettes...). On doit donc prélever aussi loin que possible des orifices d'évacuation et, dans le meilleur des cas, interrompre les rejets peu avant l'arrivée en station et pendant le prélèvement.

\section{Résultats experimentaux}

Le composé formé au cours de la réaction présente un maximum d'absorption à $526 \mathrm{~nm}$ (fig. 3). Nakas et Litchfield (1977), utilisant une méthode à la thiosemicarbazide, trouvent le maximum à $527 \mathrm{~nm}$. Il semble que les modifications du milieu réactionnel, par rapport à la méthode de Newell et al. (1967), n'aient pas eu une grande influence sur la longueur d'onde du maximum d'absorption, habituellement considéré comme étant a $520 \mathrm{~nm}$. Dans le cas présent les mesures ont été réalisées avec un filtre interférentiel Technicon CPK
$520 \mathrm{~nm}$.

\section{SENSIBILITÉ ET PRÉCISION}

Pour un réglage du colorimètre Technicon sur "standard calibration 8,0 ", la déviation totale de l'enregistreur permet l'analyse de la gamme 0 à $3.5 \mu$ atg- $\mathrm{N}$-urée/L. La réponse correspond à 0.044 unité d'absorbance pour 1 ratg-N-urée/L et un trajet optique de $50 \mathrm{~mm}$.

La réponse est linéaire de zéro à environ $15 \mu \mathrm{atg}-\mathrm{N}$-urée/L (fig. 4).

L'absence de dérive ou d'oscillation de la ligne de base entraîne une bonne reproductibilité (la fig. 5 montre un enregistrement typique). L'analyse en double de 71 échantillons d'eau de mer dont les concentrations étaient comprises entre 0 et $1 \mu$ atg- $N$-urée/ $L$ a donné un écart maximum de 0.03 et un écart-type de $0,009 \mu \mathrm{atg}-\mathrm{N}$-urée/ $\mathrm{L}$ (calculé selon la relation $\left.s=\left[(1 / 2 n) \cdot \sum_{0}^{n}\left(x_{i 1}-x_{i 2}\right)^{2}\right]^{1 / 2}\right)$.

La limite de détection est estimée à deux écarts-types, soit $0,02 \mu$ atg-N-urée/L.

\section{INTERFERENCES}

Salinité - L'analyse d'étalons réalisés en eau distillée et dans des eaux de salinité variable montre qu'il n'y a aucun effet de sel sur la réaction de l'urée avec la diacétylmonoxime entre zéro et $35 \%$ de salinité (tableau 1). L'augmentation de la salinité par addition de chlorure de sodium jusqu'à $70 \%$ semble accroitre légèrement la coloration mais de façon à peine sensible. 
TABleau 1. Influence de la salinité sur l'analyse d'un étalon à 2 ratg-N-urée/L (les mesures sont rapportées à l'eau de mer de salinité $35 \%$ ).

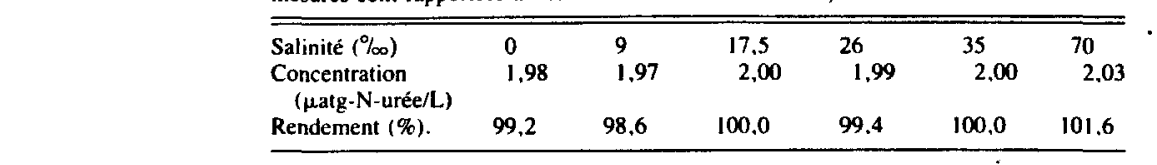

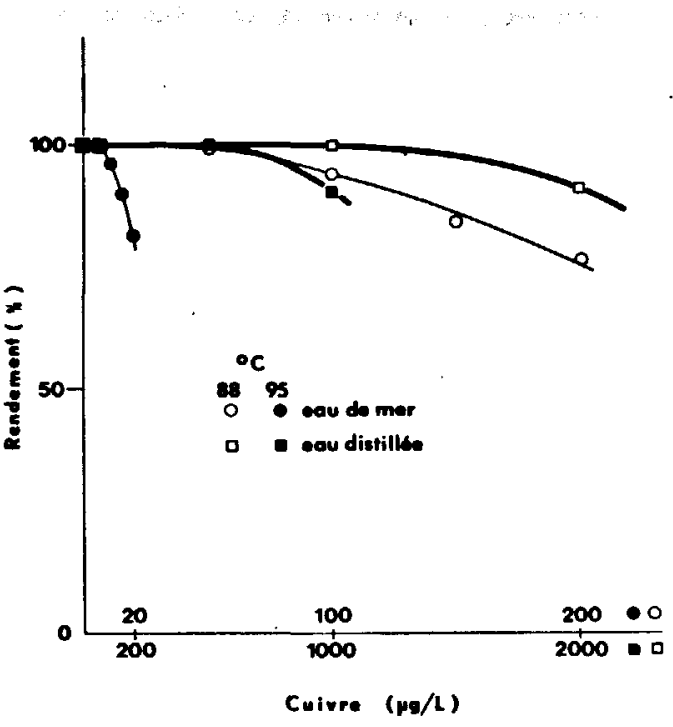

Fig 6. Influence du cuivre sur la réaction de l'urée avec la diacétylmonoxıme : à 88 et $95^{\circ} \mathrm{C}$ en eau distillée $(\square$. $\square$ ) et en eau de mer $(\mathrm{C} . \mathrm{O})$.

Ions métalliques - Ceriotti et Spandrio (1965) ont étudié l'effet d'ions métalliques dans le dosage de l'urée par la diacétylmonoxime et la phénazone dans les liquides biologıques. Sur les onze ions métalliques testés, seul $\mathrm{Cu}^{2+}$ inhibait la réaction alors que les autres la catalysarent, en particulter Fe" . Nous avons donc testé l"interference du cuiv re sur des étalons préparés d'une part en eau de mer. d'autre part en eau distillée. Comme de nombreux bains-marie ont une température fixe de $95^{\circ} \mathrm{C}$. nous avons jugé utile de contrôler cette interférence à cette température ainsi qu'à notre température de réaction $\left(88^{\circ} \mathrm{C}\right.$ ). On constate (fig. 6) qu'en eau de mer la réaction est particulièrement sensible à la présence de cuivre alors qu'elle l'est beaucoup moins en eau distillée. De plus la température joue un rôle très important sur ce phénomène qui se traduit par une baisse de sensibilité de $4 \%$ à $95^{\circ} \mathrm{C}$ pour un ajout de $10 \mu \mathrm{g} / \mathrm{L}$ dans de l'eau de mer non polluée. tandis qu'il faut ajouter environ $90 \mu \mathrm{g} / \mathrm{L}$ de $\mathrm{Cu}^{2+}$ pour obtenir le même effet à $88^{\circ} \mathrm{C}$.

Dans les conditions opératoires décrites, le risque d'inhibition est éliminé pour des eaux naturelles mêmes polluées par des éléments métalliques. Toutefois, dans certains cas particuliers où les teneurs en cuivre pourraient perturber le dosage (en particulier à $95^{\circ} \mathrm{C}$ ), on peut introduire dans le circuit de pompage de l'échantillon, avant additıon des réactifs. une petite colonne complexante de résine Chelex-100, forme $\mathrm{Na}{ }^{*}$
TABlead 2. Interférence de quelques composés dans le dosage de l'urée (les valeurs entre parenthèses ont été mesurées à $95^{\circ} \mathrm{C}$ )

\begin{tabular}{lcc}
\hline \hline & \multicolumn{2}{c}{ Interférence en \% par rapport à l'urée } \\
\cline { 2 - 3 } \multicolumn{1}{c}{ Composé } & en mole & en azote \\
\hline Uracile & 0,1 & 0,1 \\
Acide urique & 0,2 & 0,1 \\
Malonylurée & 0.1 & 0,1 \\
Thiourée & $0,8(1,6)$ & $0,8(1,6)$ \\
Allantoine & $7(12)$ & $3,5(6)$ \\
Acide carbarnylaspartique & 11 & 7 \\
Biurée & $21(30)$ & $10,5(15)$ \\
Citrulline & $112(110)$ & $75(73)$ \\
\hline
\end{tabular}

$(0,2 \mathrm{~cm} \times 5 \mathrm{~cm})$. L'introduction de cette colonne ne diminue pas la qualité des analyses et l'urée n'y est pas retenue. Cette colonne a une capacité d'échange qui permet de doser l'urée dans l'eau de mer, sans interférence du cuivre, dans une centaine d'échantillons dopés à $85 \mu \mathrm{g} / \mathrm{L}$ de $\mathrm{Cu}^{2+}$. L'efficacité de la rétention est contrôlée à l'aide d'étalons dopés et non dopés en $\mathrm{Cu}^{2+}$. La régénération de la résine $s^{*}$ effectue par pompage d'acide chlorhydrique $1 \mathrm{~mol} / \mathrm{L}$ à la place d'un échantillon. puis rinçage par une dizaine d'échantillons pauvres en cuivre.

Molécules organiques - Les interférences des acides aminés sont négligeables, comme dans la méthode de Newell et al. (1967) selon Remsen (1971). Les colorations produites par la glycıne, I'omithine, l'acide aspartique, l'acıde glutamique, la tyrosine et l'histidine sont en effet inférieures à $0,1 \%$ de celle produite par l'urée. L'ammonium n'interfère pas non plus. Le tableau 2 rassemble les résultats obtenus avec les composés interferant à $0,1 \%$ ct plus. Trois composés interfèrent légèrement : I'allantoine $(3.5 \%)$. l'acide carbamylaspartıque $(7 \%)$ et la biurée $(10.5 \%)$. Scule la citrulline interfere de façon importante, paraissant se comporter stoechıométriquement comme l'urée dans la réactıon, mais avec formation d'une coloration légèrement plus intense (112\%).

Cependant, en pratique, un composé ne pourra être considéré comme une réelle interférence que si sa concentration dans les milieux étudiés est suffisante pour donner un signal non nćgligeable par rapport à l'urée. Ce n'est pas le cas de 1'allantoine (Remsen 1971), ni de l'acide carbamylaspartique, composé entrant dans le cycle de synthèse des bases nucléiques. Quant à la biurée, il ne s'agit pas d'un composé d'origine biologique et sa présence dans l'eau de mer paraît extrèmement improbable.

En revanche. Remsen (1971) n'exclut pas la possibilité de la présence de citrulline dans les eaux riches en zooplancton. Afin de vérifier ce poınt. nous avons développé un procédé de détermination de la citrulline par différence, en analysant les 
TABleaU 3. Résultats des contrôles de conservation des échantillons.

\begin{tabular}{|c|c|c|c|}
\hline Durée & $\begin{array}{c}\text { Nombre } \\
\text { d'èchantillons }\end{array}$ & $\begin{array}{l}\text { Concentrations } \\
\mu \text { atg- } N \text {-urée/L }\end{array}$ & 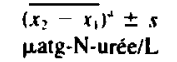 \\
\hline $\begin{array}{l}3 \text { semaines } \\
16 \text { mois }\end{array}$ & $\begin{array}{l}17 \\
26\end{array}$ & $\begin{array}{r}0,1-2 \\
0.04-3\end{array}$ & $\begin{aligned}+0.014 & \pm 0.017 \\
-0.05 & \pm 0.06\end{aligned}$ \\
\hline
\end{tabular}

$\left(\widehat{x_{2}-x_{1}}\right)^{2}:$ moyenne des différences entre les mesures sur le mème échantillon, avant et après la phase de congélation.

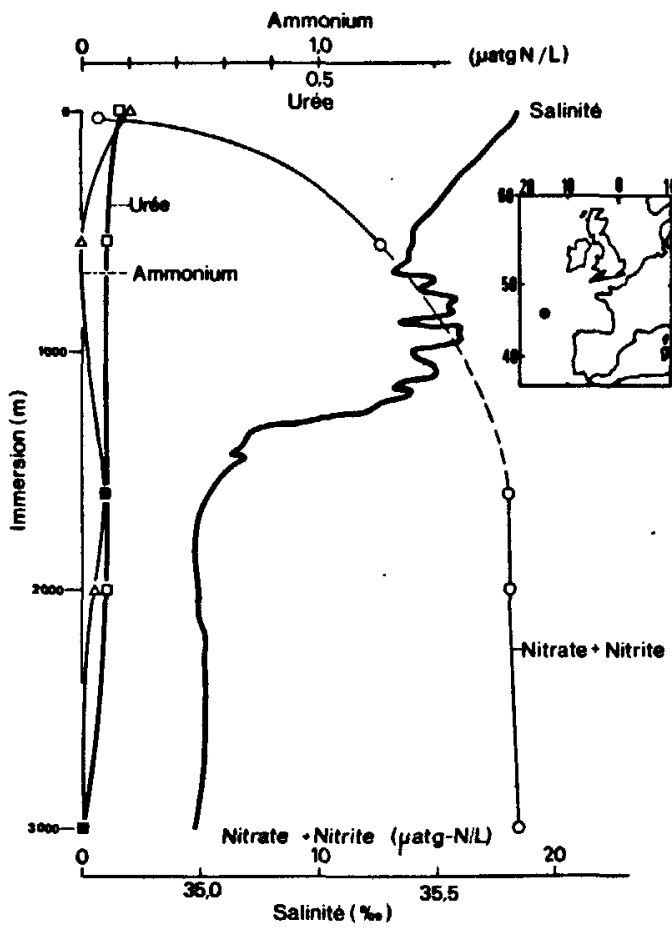

FiG. 7. Répartition verticale de la salinité, des ions nitrate + nitrite, de l'ammonium et de l'urée dans le golfe de Gascogne $\left(46^{\circ} 42^{\prime} \mathrm{N}\right.$ : $14^{\circ} 41.4^{\prime} \mathrm{W}$ ).

échantillons avant et après élimination de ce composé interférant. Dans ce but, les échantillons sont passés sur une colonne complexante de Chelex-100-Cu ${ }^{2+}$ où la citrulline est retenue grâce à sa fonction acide aminé (Siegel et Degens 1966). La colonne de Chelex $-\mathrm{Cu}^{2+}$ de $3 \mathrm{~cm}^{3}(0,8 \mathrm{~cm} \times$ $6 \mathrm{~cm}$ ) est préparée par percolation sur une colonne de Chelex$\mathrm{Na}^{+}$d'une solution ammoniacale de cuivre $\left(\mathrm{NH}_{3}: 3 \mathrm{~mol} / \mathrm{L}\right.$; $\mathrm{Cu}^{2+}: 0.5 \mathrm{~mol} / \mathrm{L}$ ), puis rinçages à l'ammoniaque $3 \mathrm{~mol} / \mathrm{L}$ et à l'eau distillée. Une colonne de $2 \mathrm{~cm}^{3}$ de Chelex- $\mathrm{Na}^{+}$est mise en série pour retenir le cuivre perdu par la Chelex- $\mathrm{Cu}^{2}$ au passage de l'eau de mer.

Une colonne fraîchement préparée n'est utilisée que pour une vingtaine d'échantillons de $20 \mathrm{~mL}$ afin d'éliminer le risque de saturation (la résine retenant de nombreux composés complexant le $\mathrm{Cu}^{2+}$ ). Le rendement est contrôlé à l'aide

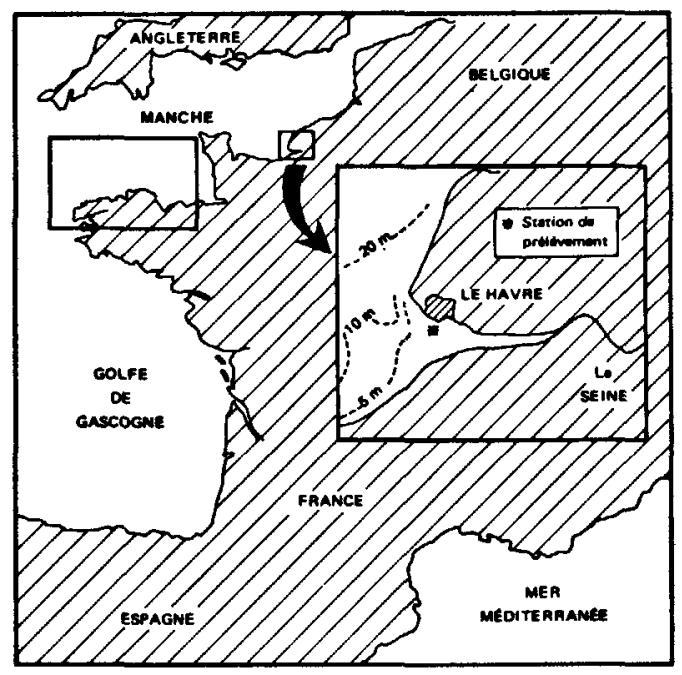

FIG. 8. Localisation des zones côtière et cstuarienne où les concentrations d'urée ont été analysées.

d'étalons passés après chaque série d'échantillons : le passage dans cet ordre évite la migration dans la colonne et la contamination éventuelle des échantillons. dont les concentrations sont en principe faibles. Dans ces conditions. la rétention est de $100 \%$ pour la citrulline et de $0 \%$ pour l'urée.

Nous avons ainsi analysé 23 échantillons d'eau de mer filtrée, prélevés près des côtes de la Manche. contenant de 0.05 à $1.6 \mu \mathrm{atg}-\mathrm{N}$-urée/L. La baisse moyenne de concentration après passage sur la colonne a été de $0.007 \mu$ atg-Nurée/L. tandis que les écarts constatés sur le même échantillon. entre les mesures avant et après passage sur la colonne. étaient compris dans la fourchette $\pm 0.05 \mu \mathrm{atg}-\mathrm{N}$-urée $/ \mathrm{L}$ (dont 21 sur 23 dans la fourchette $\pm 0.03 \mu$ atg- $N$-urée/L). Ces écarts ne sont en aucun cas significatifs et correspondent à la précision que l'on peut attendre de ce double dosage dans les conditions opératoires décrites.

Il n'a donc pas été possible de mettre en évidence la présence de citrulline dans les eaux analysées. alors que 20 des échantillons analysés provenaient de la Baie de Seine (mai 1978) où l'on comptait de 1500 à 5000 individus zooplanctoniques par mètre cube (Le Fèvre-Lehoërff 1980).

\section{Application}

La méthode ci-dessus décrite est utilisée en routine pour l'analyse d'eaux marines et estuariennes.

Les prélèvements sont effectués à l'aide de bouteilles Niskin en PVC et l'échantillonnage se fait dans des flacons en polyéthylène ou polypropylène de $125 \mathrm{~mL}$. Ces flacons sont traités à l'acide chlorhydrique $1 \mathrm{~mol} / \mathrm{L}$ avant usage. Au remplissage, l'eau est filtrée directement sur un filet à $50 \mu \mathrm{m}$ de façon à éliminer le zooplancton et les grosses particules minérales. Ce filet est inséré dans un dispositif de filtration en continu que l'on branche directement à la bouteille de prélèvement. procédure qui permet d'éviter des contaminations. 


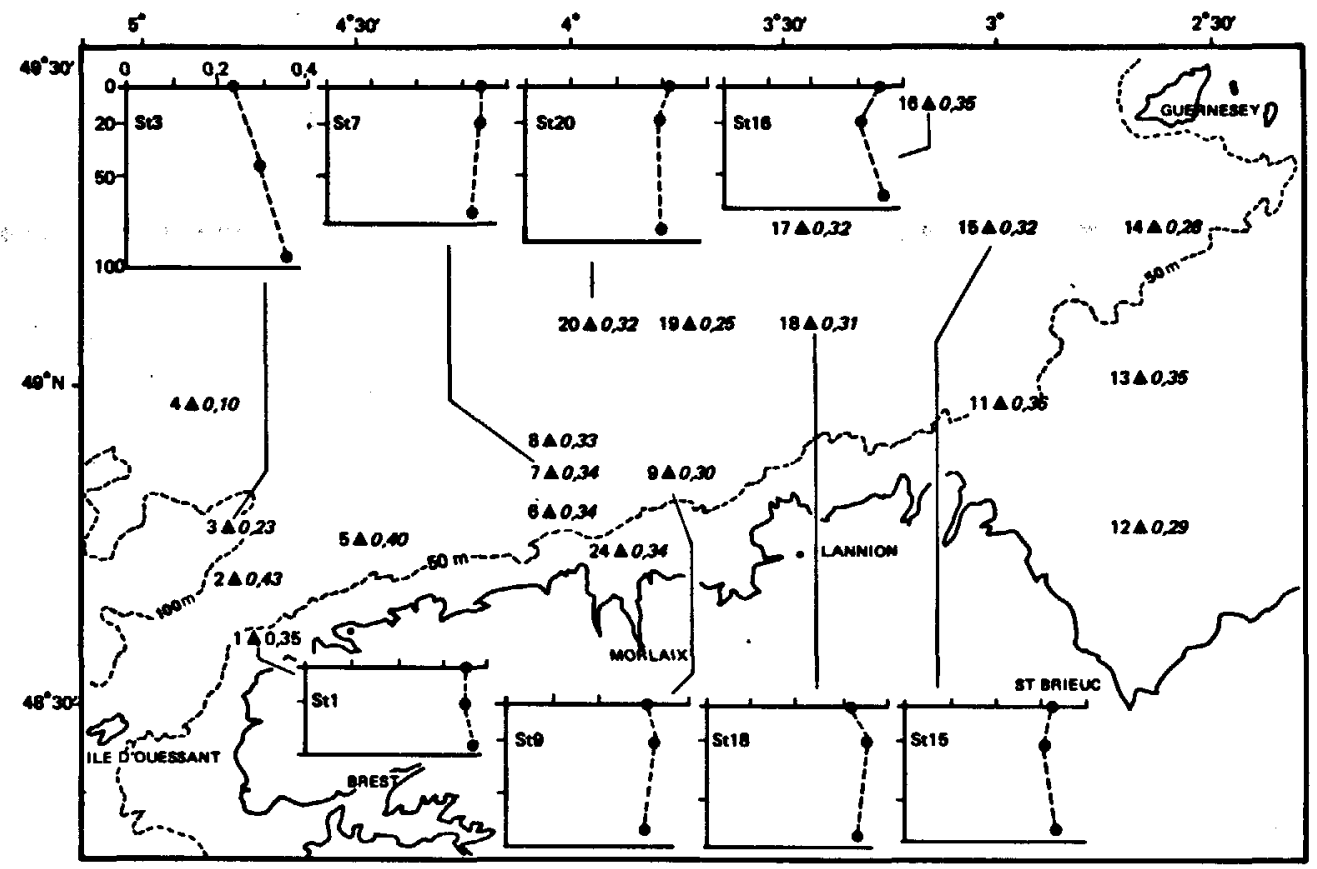

Fig 9. Concentrations d'urée ( $\mu$ atg-N-urée/ $L$ ) en Manche Sud-Occidentale dans la pénode du 30 mars au 2 avrıl 1978. Les numéros des stations sont indiqués à gauche du symbole reperant leurs positions $(\mathbf{A})$.

Les échantillons ne pouvant pas toujours être analysés très peu de temps après le prélèvement, il a été nécessaire de les placer au congẻlateur à $-20 /-25^{\circ} \mathrm{C}$ aussitôt après l'échantillonnage. Nous avons vérifié la validité de cette procédure à court terme ( 3 semaines) et à long terme ( 16 mois) avec des échantillons d'eaux côtières et estuariennes. Pour l'essai à court terme. 17 échantillons ont été analysés peu après le prélèvement, immédiatement avant leur mise au congélateur. l.'cssai à long terme a été effectué sur une autre série de 26 échantillons, analysés une première fois une à deux semaines après le prélèvement. replacés aussitôt au congélateur puis analysés à nouveau 16 mo plus tard. Les résultats de ces contròles sont rassemblés dans le tableau 3. Au bout de trois semaines on ne note aucune différence significative entre les échantillons, tandis quaprès $16 \mathrm{mo}$, une légère diminutıon de concentration paraît avoir en lieu $(0,05 \mu$ atg- $N$-urée $/ L)$.

En application de la méthode proposée nous présentons ci-dessous des résultats acquis en trois zones marines différentes (oceanıque, côtière et estuarienne).

La fig. 7 représente un profil dans l'Atlantique Nord à environ 350 milles des côtes européennes. Les concentrations entre la surface et $3000 \mathrm{~m}$ sont inférieures à $0,08 \mu \mathrm{atg}-\mathrm{N}$ urée/L. À $3000 \mathrm{~m}$. il y a épuisement total de l'urée ainsi que de l'ammonium qui reste également à des valeurs très basses aux autres niveaux (maxımum $0.20 \mu$ atg- $\mathrm{N}-\mathrm{NH}_{4}^{+} / \mathrm{L}$ en surface). Les concentrations en nitrate $(+$ nitrite), croissant avec la profondeur tout d'abord rapidement. puis de façon très lente à partir de $1500 \mathrm{~m}$, présentent une évolution océanique classique.
Les deux sites côtier et estuarien étudiés sont indiqués sur la fig. 8. La zone marine côtière est la Manche Sud-Occidentale où 20 stations ont été échantillonnécs du 30 mars au 2 avril 1978. Les résultats sont présentés sur la fig. 9. Les concentrations sont comprises entre 0,10 et $0,43 \mu$ atg $\mathrm{N}$ urée/L, la moyenne en surface étant de $0.31 \mu$ atg-N-urée/ $L$. On note une certaine homogénéité horizontale et verticale. cette dernière s'expliquant par l'absence de stratıfication due à l'intenutte des courants de marée. Les concentrations les plus basses se situent aux stations 3 et 4 . les plus à l'ouest : le développement phytoplanctonique mis en évidence dans cette zone par d'autres mesures telles que les éléments nutritifs mineraux (N. P. Si), la chlorophylle et l'oxygene dissous (Amınot et Kérouel 1978: Aminot 1981), pourrait également être responsable de la consommation d'urée.

Les résultats présentés sur la fig. 10 ont été obtenus au cours d'un cycle de marée effectué à point fixe le 5 octubre 1978 dans lestuaire de la Seine en face du Port du Havre (France). Des prélèvement ont été effectués en surface et au fond. Dans cette zone la salinité descend à près de $23 \%$ à basse mer tandis que les concentrations en urée varient de 0.86 à $1,80 \mu$ atg- $N$-urée/L. On notera que l'urée varie en sens inverse de la salinité. le plus fortes valeurs se trouvant donc aux environs de la basse mer.

\section{Discussion}

Dans la méthode de dosage de l'urée que nous présentons, nous avons pu éliminer une grande partie des additifs utilisés 


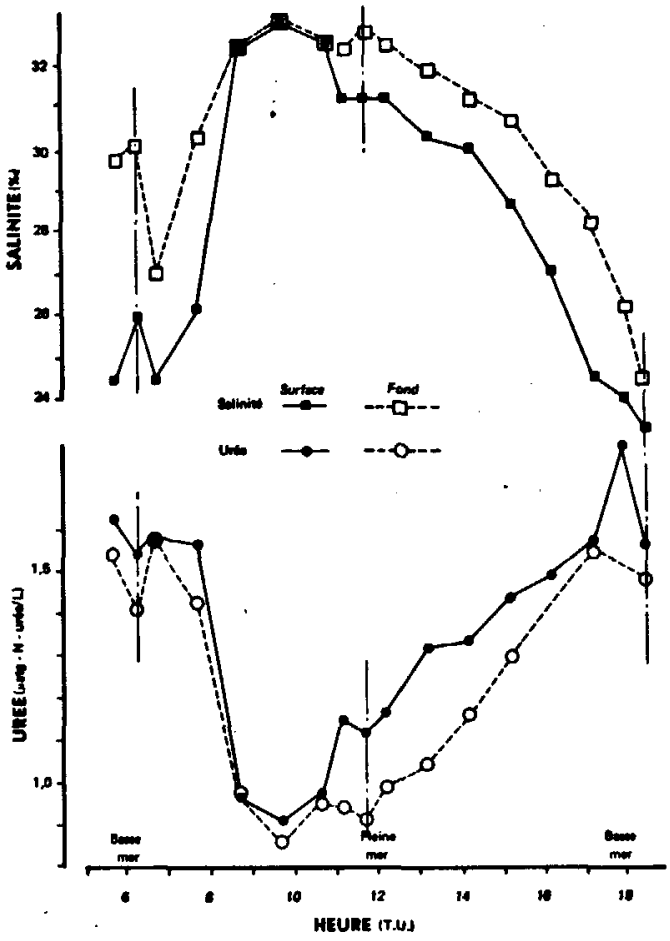

FiG. 10. Evolution de la concentration d'urée (surface : 0 . fond O) et de la salinité (surface : $\square$, fond: $\square$ ), dans l'estuaire de la Seine, au cours d'un cycle de marée le 5 octobre 1978.

par les auteurs précedents pour stabiliser la coloration. L'introduction de la thiosemicarbazide est essentiellement à l'origine de ce fait; toutefois, l'accroissement de sensibilité obtenu avec ce composé ne s'explique pas simplement. Le colorant formé absorbe à une longueur d'onde très voisine des colorants obtenus avec la semicarbazide et les additifs complexes introduits par les précédents auteurs et peut donc présenter une structure comparable. L'ion $\mathrm{Fe}^{3+}$ a un effet catalytique évident qui n'est pas aussi intense avec d'autres métaux (Mn, Sb). En éliminant le chlorure de sodium, précédemment introduit dans la quasi totalité des modes opératoires décrits antérieurement, on évite une dilution supplémentaire de l'échantillon. La suppression des jons nitrate. phosphate et manganèse, également introduits dans la majorité des processus, simplifie considérablement la préparation des réactifs. Finalement, en combinant les débits et les concentrations des réactifs, nous avons pu accroître très nettement la sensibilité (environ $\times$ 2,5 par rapport à la méthode de DeManche et al. 1973).

Nous avons mis en évidence que la diminution de la température de réaction de 95 à $88^{\circ} \mathrm{C}$ présentait de nombreux avantages. D'une part, la qualité des mesures s'est améliorée par une meilleure hydraulique dans le circuit analytique et une diminution importante des blancs; d'autre part, la spécificité s'est accrue par réduction des interférences de molécules organiques. En outre, la réduction de l'inhibition du cuivre permet d'appliquer cette méthode dans la plupart des milieux aqueux quel que soit leur degré de pollution.

En ce qui concerne l'obtention d'eaux de mer de référence. et, par conséquent, la détermination du blanc d'effet de sel, nous pensons qu'il était important de définir une méthode simple pour y parvenir. Seule la détermination rigoureuse des blancs permet en effet de mesurer de façon aussi exacte que possible des concentrations dans le milieu. Plusieurs méthodes peuvent être envisagées pour y parvenir. Comme l'indiquent DeManche et al. (1973), l'utilisation d'eau de mer artificielle donne des blancs erronés en raison de la présence d'urée dans les sels utilisés. Ces auteurs réduisent donc chimiquement la sensibilité pour mesurer la blanc d'effet de sel. Nous avons montré que cette technique ne donnait pas satisfaction et que seule l'utilisation d'eau de mer exempte d'urée était envisageable pour mesurer rigoureusement le blanc dans les conditions normales de réaction.

Pour ce faire, nous avons testé divers procédés. La destruction enzymatique de l'urée selon la méthode de McCarthy (1970) ne peut être utilisée car la présence d'uréase perturbe la réaction chimique à la diacétylmonoxime. L'irradiation par U.V. de l'eau de mer, selon Armstrong et al. (1966) (lampe Original Hanau de $900 \mathrm{~W}$ : durée d'irradiation $40 \mathrm{~h}$ ), n'a pas permis d'obtenir des blancs suffisamment bas au pH de l'eau de mer. tandis qu'en milieu acide le chlore formé perturbe le dosage. Finalement, la solution adoptée, c'est-à-dire le passage sur charbon actif d'une eau de mer quasiment épuisée; $s$ "avère simple et rigoureuse comme nous avons pu le vérifier par comparaison avec les analyses d'eaux de mer profondes.

Du point de vue de la spécificité, nous pensons que celle-ci est tout à fait satisfaisante si l'on considère les molécules qui interfèrent et leur degré d'interférence. Seule la citrulline pourrait réellement interférer dans le dosage si l'on en trouvait dans l'eau de mer en quantité suffisante. Or les déterminations que nous avons effectuées sur ce composé dans des eaux côtières assez riches en zooplancton n'ont pas permis de la mettre en évidence. Macko et Green (1979), qui ont analysé des acides aminés dans un estuaire, n'ont pas non plus détecté de citrulline. Nous pensons donc que le dosage colorimétrique de l'urée tel que nous le proposons est, en pratique, spécifique de ce composé dans le milieu marin. En outre, pour les cas très particuliers où la présence de citrulline ne serait pas à exclure, on peut doser spécifiquement ce composé comme nous l'avons fait.

D'une façon générale, la sensibilité et la reproductibilité du dosage de l'urée décrit ici nous permet de considérer qu'il est préférable à la méthode enzymatique de McCarthy (1970). En effet, cette dernière méthode nécessite d'effectuer deux mesures de concentrations en ammonium, paramètre dont le dosage présente en lui-mème une précision moins bonne que celle du présent dosage de l'urée. En outre, dans la méthode enzymatique, les échantillons doivent être préservés à la fois contre les risques de contamination en ammonium et en urée.

La méthode a été appliquée avec succès à diverses eaux marines et estuariennes : les concentrations trouvées au large sont inférieures à $0.1 \mu \mathrm{atg}-\mathrm{N}$-urée $/ \mathrm{L}$, tandis que près des côtes françaises. elles sont de l'ordre de 0,3 à $0,4 \mu$ atg-Nurée/L et s'élèvent jusqu'à $1,8 \mu \mathrm{atg}-\mathrm{N}$-urée/L en estuaire à la salinité de $23 \%$. Ces résultats ont comparables à ceux mentionnées par Remsen et al. (1974) et confirment l'influence des apports continentaux sur les concentrations en urée dans 
les eaux marines.

\section{Remerciements}

Nous remercions A. Vangriesheim. A. Billant et J. P. Gouillou du Département Etudes Océaniques du Centre Océanologique de Bretagne pour les prélèvements et les mesures de salinité du Golfe de Gascogne.

AmINoT. A. 1981. Anomalies du système hydrobiologique côtier après l'échouage de l'Amoco Cadiz. Considérations qualitatives et quantitatives sur la biodégradation in situ des hydrocarbures. p. 223-242. Dans Amoco Cadiz. Conséquence d'une pollution accidentelle par les hydrocarbures. CNEXO. Paris.

Aminot. A.. et R. Kerouel. 1978. Premiers résultats sur l'hydro- . logie, l'oxygène dissous et les pigments photosynthétiques en Manche Occidentale après l'échouage de l'Amoco Cadiz. Publications du CNEXO. Actes de Colloques. 6: 51-68.

Antia, N. J., B. R. Berland, D. J. Bonin, and S. Y. Maestrini, 1977. Effects of urea concentration in supporting growth of certain marine microplanktonic algae. Phycologia 16: 105-111.

armstrong, F. A. J., P. M. Williams, and J. D. H. Strickland. 1966. Photo-oxidation of organic matter in sea water by ultraviolet radiation. analytical and other applications. Nature 211: $481-483$.

Beale, R. N., and D. Croft. 1961. A sensitive method for the colorimetric determination of urea. J. Clin. Pathol. 14: $418-424$

Carpenter, E. 3., C. C. Remsen, and B. W. Schroeder. 1972. Comparison of laboratory and in situ measurements of urea decomposition by a marine diatom. J. Exp. Mar. Biol. Ecol. 8: $259-264$

Ceriotti. G., and L. SPandrio. 1963. A spectrophotometric method for determination of urea. Clin. Chim. Acta 8: 295-299.

1965. Catalytic acceleration of the urea-diacetylmonoximephenazone reaction and its application to automatic analysis. Clin. Chim. Acta 11: 519-522.

Corner, E. D. S.. AND B. S. Newell. 1967. On the nutrition and metabolism of zooptankton. J. Mar. Biol. Assoc. U.K. 47: $113-120$.

Coulombe, J. J.. and L. Favreau. 1963. A new simple semimicro method for colorimetric determination of urea. Clin. Chem. 9: 102.

DeManche. J. M. . H. Curl JR.. and D. D. Coujghenower. 1973. An automated analysis for urea in sea water. L.mnol. Oceanogr. 18: $686-689$.

Dugdale. R. C., and J. J. Gofring. 1967. Uptake of new and regenerated forms of nitrogen in primary productivity. Limnol. Oceanogr. 12: 196-206.

EMMET, R. T. 1969. Spectrophotometric determination of urea and ammonia in natural waters with hypochlorite and phenol. Anal. Chem. 41: 1648-1652.

Eppley, R. W., A. F. Carlucci, O. Holm-Hansen. D. Kiefer. J. J. MCCarthy, E. Venrick, and P. M. Williams. 1971. Phytoplankton growth and composition in shipboard cultures supplied with nitrate. ammonium, or urea as the nitrogen source. Limnol. Oceanogr. 16: 741-75।

Fearon, W. R. 1939. The carbamido diacetyl reaction: a lest for citrulline. Biochem. J. 33: 902-907

Froelich. P. N. AND M. E. Q. PILson. 1978. Systematic absorbance errors with Technicon Autoanalyzer II colorimeters. Water Res. 12: $599-603$.

Grasshofr, K. 1976. Procedures for the automatic determination of seawater constituents, p. 276-289. In K. Grasshoff [ed.] Methods of seawater analysis . Verlag Chemie. Weinheim. New York.

KorOLEFF. F. 1976. Determination of urea. p. 145-149. In K. Grasshoff [ed.] Methods of seawater analysis. Verlag Chemie, Weinheim, New York.

Le Corre. P. et P. Tréguer. 1978. Dosage de l'ammonium dans l'eau de mer : comparaison entre deux méthodes d analyse automatique. J. Cons, Int. Explor. Mer 38: 147-153.

Le Fèvre-Lehoerff, G. 1980. Le zooplancton de la baie de Seine. p. 129-151. Dans Baie de Seine, Campagne Thalia mai 1978. présentation des résultats. Département ELGMM, Centre Océanologique de Bretagne. BP 337, 29273 Brest Cedex France.

Macko, S. A., ANo E. J. Green. 1979. The quantification of amino acids in seawater using thin-layer chromatography and fluorimetry. Mar. Chem 8: 181-189.

Marsh, W. H.. B. Fingerhut, AND H. Miller. 1965. Automated and manual direct methods for the determination of blood urea. Clin. Chem. 2: 624-627.

Marshall. E. K., JR. 1913. A rapid clinical method for the estimation of urea in urine. J. Biol. Chem. 14: 283-290.

Mauzac, M. F. Guerard, J. Mathieu. et J. Laroche. 1976. Dosage du formaldéhyde. de l'urée et des détergents anioniques dans les effluents d'une usine de produits chimiques. Analusis 4: $236-245$

McCaRthy, J. J. 1970. A urease method for urea in sea water. Limnol. Oceanogr. 15: 309-313.

1972. The uptake of urea by natural populations of marine phytoplankton. Limnol. Oceanogr. 17: 738-748.

NAKAS, J. P., AND C. D. Litchfield. 1977. Application of the diacetylmonoxime thiosemicarbazide method to the analysis of urea in estuarine sediments. Estuar. Coast. Mar. Sci. 5: $143-150$.

Newell, B. S., B. Morgan, AND J. Cundy. 1967. The determination of urea in sea water. J. Mar. Res. 25: 201-202.

Prescott. L. M., and M. E. Jones. 1969. Modified methods for the determination of carbamyl aspartate. Anal. Biochem. 32: $408-419$.

Remsen, C. C. 1971. The distribution of urea in coastat and oceanic waters. Limnol. Oceanogr. 16: 732-740.

Remsen, C. C., E. J. Carpenter, and B. W. Schroeder. 1974. The role of urea in marine microbial ecology. p. 286-304. In R. R. Colwell and R. Y. Morita [ed.], Effects of the ocean environment on microbial activities. University Park Press, Baltimore, London, Tokyo.

Siegel, A., ANd E. T. Degens. 1966. Concentration of dissolved amino acids from saline waters by ligand-exchange chromatography. Science 151: 1098-1101.

StRickLAND, J. D. H., AND T. R. PARsons. 1972. A practical handbook of sea water analysis. Bull. Fish. Res. Board Can. 167: $310 \mathrm{p}$.

WheATLEY, J. R. 1948. An improved diacetyl reaction for the estimation of urea in blood. Biochem. J, 42: xli-xlii. 